\title{
Poor Respiratory Health Following Relapsing SARS-CoV-2 Infection in Children with Cystic Fibrosis
}

\author{
Nitin Dhochak ${ }^{1} \cdot$ Kana Ram Jat $^{1} \cdot$ Kapil Dev Soni ${ }^{2} \cdot$ Yudhyavir Singh $^{2} \cdot$ Richa Aggarwal $^{2} \cdot$ Rakesh Lodha $^{1} \cdot$ \\ Anjan Trikha ${ }^{2} \cdot$ Sushil K. Kabra ${ }^{1}$
}

Received: 3 August 2021 / Accepted: 25 October 2021 /Published online: 28 January 2022

(c) Dr. K C Chaudhuri Foundation 2022

\begin{abstract}
Children with cystic fibrosis (CF) constitute a high-risk group for COVID-19 with underlying chronic lung disease. COVID19 severity varying from mild infection to need of intensive care has been described in children with CF. Two children with significant underlying pulmonary morbidity are described here, who developed severe disease following SARS-CoV-2 infection. Case 1 (a 9-y-old boy) had pneumonia with respiratory failure requiring noninvasive ventilation support. He had delayed clearance of SARS-CoV-2, with recurrence of symptomatic disease with short asymptomatic period in between. He was also diagnosed with CF-related diabetes and allergic bronchopulmonary aspergillosis during the second episode. Case 2 (an 18-mo-old boy) had two episodes of SARS-CoV-2-related severe lower respiratory infection within a period of 2 mo, requiring high-flow nasal oxygen support. Both children had $3^{\text {rd }}$ pulmonary exacerbation but SARS-CoV-2 was not detected in respiratory secretions. To conclude, children with CF with underlying pulmonary morbidity, can develop severe COVID-19 and prolonged SARS-CoV-2 shedding.
\end{abstract}

Keywords Cystic fibrosis $\cdot$ SARS-CoV-2 $\cdot$ Poor lung health $\cdot$ Prolonged viral shedding

\section{Introduction}

Children with cystic fibrosis (CF)-related chronic lung disease are predisposed to severe pulmonary infections with severe acute respiratory syndrome coronavirus-2 (SARS-CoV-2). Though initial reports demonstrated asymptomatic and mild SARS-CoV-2 infection in children with CF, larger series have described all degrees of severity including intensive care admission especially in post-transplant patients [1-3]. Children with $\mathrm{CF}$ from developing countries frequently have advanced lung disease [4]. The authors' unit runs a weekly CF clinic with nearly 200 children in regular follow-up cared by clinical specialists, physiotherapist, nutritionist, and specialist nurse. Presentation of two children with CF with COVID19 are described here.

Rakesh Lodha

rlodha1661@gmail.com

1 Department of Pediatrics, All India Institute of Medical Sciences, New Delhi 110029, India

2 Department of Anaesthesiology, Pain Medicine and Critical Care, All India Institute of Medical Sciences, New Delhi, India

\section{Case 1}

A 9-y-old boy (homozygous F508del, Staphylococcus aureus colonization, BMI -2.18 z-score, CF-related liver disease, on regular azithromycin) had no pulmonary exacerbation in the last 11 mo. He developed fever, increased cough, and fast breathing for $3 \mathrm{~d}$. He received intravenous piperacillin-tazobactam and amikacin for $14 \mathrm{~d}$, but did not improve. At presentation to the authors' hospital, respiratory specimen was positive for SARS-CoV-2 reverse-transcriptase polymerase chain reaction (RT-PCR). He required noninvasive ventilation for $2 \mathrm{~d}$, followed by high-flow nasal canula (HFNC) support. Inflammatory markers showed no significant abnormalities (Supplementary Table S1). Chest radiograph showed hyperinflation, mucous plugging, and interstitial shadows (Fig. 1a). As per the institutional protocol, he received remdesivir ( $5 \mathrm{~d})$, hydrocortisone, and cefoperazone-sulbactam and vancomycin for $14 \mathrm{~d}$. Child became afebrile on day 8 of admission. He was discharged after $14 \mathrm{~d}$ while still SARS-CoV-2 RT-PCR positive.

Within $4 \mathrm{~d}$ of discharge, he again developed intermittent fever spikes and increased cough. But he was brought to the authors'center with breathing difficulty and persistent 

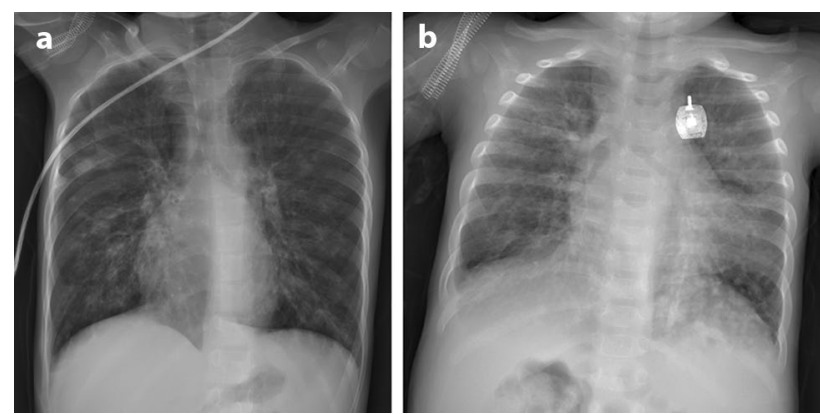

Fig. 1 Chest radiograph of (a) case 1 and (b) case 2 at the time of first admission showing interstitial infiltrates with background chronic lung disease changes

fever after 20 d. His SARS-CoV-2 RT-PCR was still positive. He received oxygen by facemask and intravenous antibiotics. Sputum culture grew E. coli. He was also diagnosed with CF-related diabetes (CFRD) and allergic bronchopulmonary aspergillosis during this admission (Supplementary Table S1). SARS-CoV-2 RT-PCR turned negative by day 10 of hospital stay.

He had another pulmonary exacerbation at $3 \mathrm{mo}$, but this time, SARS-CoV-2 RT-PCR was negative. Sputum cultures grew E. coli, Klebsiella pneumoniae, and methicillin-resistant Staphylococcus aureus. He was discharged after $20 \mathrm{~d}$ of treatment.

\section{Case 2}

An 18-mo-old boy (diagnosed by elevated sweat chloride, heterozygous c.1333delA, BMI $-0.90 z$ score, on regular azithromycin) had last pulmonary exacerbation 7 mo back. He had increased cough for $3 \mathrm{~d}$, and fever, fast breathing, and desaturations for $1 \mathrm{~d}$. He had hypoxia with bilateral crepitations and wheeze. His SARS-CoV-2 RT-PCR was positive. Inflammatory markers were not elevated (Supplementary Table S1). Chest radiograph showed interstitial infiltrates (Fig. 1b). He received HFNC support for $4 \mathrm{~d}$ and oxygen was gradually tapered. He received meropenem, vancomycin, and prednisolone $(1 \mathrm{mg} / \mathrm{kg} / \mathrm{d})$. He was discharged after $14 \mathrm{~d}$ with negative SARS-CoV-2 RT-PCR.

Thirty-four days following discharge, the child received antibiotics for $10 \mathrm{~d}$ for pulmonary exacerbation locally. While on treatment, he again developed fever, worsening cough, fast breathing, and wheezing for $3 \mathrm{~d}$. He was admitted to the authors'center; respiratory sample was positive for SARS-CoV-2. He was treated with intravenous antibiotics, HFNC $(20 \mathrm{~L} / \mathrm{min})$, and prednisolone. He was discharged after $14 \mathrm{~d}$ with negative SARS-CoV-2 RT-PCR. There was no fresh SARS-CoV-2 infection in the house. IgG against SARS-CoV-2 was significantly elevated.
After 3 mo from the first episode, he again had increased cough, fast breathing, and wheezing, which was managed in the outpatient setting with oral antibiotics and a short course of prednisolone.

\section{Discussion}

Two children with CF with severe SARS-CoV-2 infection have been discussed. While the first case had persistent infection, the second might have had recurrent infection due to SARS-CoV-2. Both had frequent pulmonary exacerbations following initial COVID-19. In published literature, a significant proportion of CF patients require hospitalization (29\%) when they develop COVID-19 as compared to overall pediatric age group $(0.1 \%-2.2 \%)[1,5]$. Oxygen requirement was reported in $6 \%$ and $29 \%$ in two studies $[1,2]$. Both the cases had severe disease.

Prolonged shedding of SARS-CoV-2 up to $120 \mathrm{~d}$ has been described in immunocompromised adults [6]. The first case described here had SARS-CoV-2 RT-PCR positive till 55 $\mathrm{d}$ of onset of symptoms. The second case had one negative SARS-CoV-2 RT-PCR in between, so it is difficult to conclude if the second episode was re-infection or persistent infection. CF-related innate-immune dysfunction, use of steroids, and new-onset diabetes could cause prolonged viral shedding $[7,8]$.

Viral infection in children with CF can cause pulmonary exacerbations due to direct infection, as well as secondary bacterial infection [9]. Both of the above-mentioned cases had poor respiratory health with pulmonary exacerbations after SARS-CoV-2 infection, despite having wellcontrolled disease in the previous year. Case 1 also had new onset CFRD. New-onset type 1 diabetes has been seen with COVID-19; the presence of SARS-CoV-2 receptors on pancreatic exocrine cells, use of steroids, and infection-related stress response have been the postulated mechanisms [10].

\section{Conclusion}

Children with CF having significant lung morbidity can develop severe infection and repeated pulmonary exacerbations. Strict preventive measures should be taken to protect children with CF from SARS-CoV-2.

Supplementary Information The online version contains supplementary material available at https://doi.org/10.1007/s12098-021-04057-0.

Acknowledgements The authors are thankful to the Virology Laboratory, Department of Microbiology, All India Institute of Medical Sciences, Delhi and Indian Council of Medical Research, Delhi for laboratory support for SARS-CoV-2 RT-PCR. 
Authors' Contributions ND, KRJ, RL, and SKK were involved in conception of work, acquisition and interpretation of information, and drafting the work; KDS, YS, RA, and AT contributed by acquisition and interpretation of information, and drafting the work. RL will act as the guarantor for this paper.

\section{Declarations}

Conflict of Interest None.

\section{References}

1. Bain R, Cosgriff R, Zampoli M, et al. Clinical characteristics of SARS-CoV-2 infection in children with cystic fibrosis: An international observational study. J Cyst Fibros. 2021;20:25-30.

2. Moeller A, Thanikkel L, Duijts L, et al. COVID-19 in children with underlying chronic respiratory diseases: survey results from 174 centres. ERJ Open Res. 2020;6:00409-2020.

3. Mathew HR, Choi MY, Parkins MD, Fritzler MJ. Systematic review: cystic fibrosis in the SARS-CoV-2/COVID-19 pandemic. BMC Pulm Med. 2021;21:173.
4. Dhochak N, Jat KR, Sankar J, Lodha R, Kabra SK. Predictors of malnutrition in children with cystic fibrosis. Indian Pediatr. 2019;56:825-30.

5. Children and COVID-19: State-Level Data Report. In: American Academy of Pediatrics. 2021. Available at: http://services.aap.org/ en/pages/2019-novel-coronavirus-covid-19-infections/children-andcovid-19-state-level-data-report/. Accessed 1 March 2021.

6. Li Q, Zheng XS, Shen XR, et al. Prolonged shedding of severe acute respiratory syndrome coronavirus 2 in patients with COVID-19. Emerg Microbes Infect. 2020;9:2571-7.

7. Cohen TS, Prince A. Cystic fibrosis: a mucosal immunodeficiency syndrome. Nat Med. 2012;18:509-19.

8. Peckham D, McDermott MF, Savic S, Mehta A. COVID-19 meets cystic fibrosis: for better or worse? Genes Immun. 2020;21:260-2.

9. Gulla KM, Balaji A, Mukherjee A, et al. Course of Illness after viral infection in Indian children with cystic fibrosis. J Trop Pediatr. 2019;65:176-82.

10. Unsworth R, Wallace S, Oliver NS, et al. New-onset type 1 diabetes in children during COVID-19: multicenter regional findings in the U.K. Diabetes Care. 2020;43:e170-1.

Publisher's Note Springer Nature remains neutral with regard to jurisdictional claims in published maps and institutional affiliations. 